\title{
A Review on Identity based Cryptography
}

\author{
Sonia Thakur \\ Research Scholar, CEC Landran
}

\author{
Heena \\ Asstt.Prof. I.T. Deptt.CEC Landran
}

\begin{abstract}
Security in mobile ad-hoc networks (MANET's) continues to draw in attention when years of analysis. Recent advances in identity-based cryptography (IBC) sheds light-weight on this drawback and has become widespread as an answer base. In this research it is presenting a comprehensive image and capture the state of the art of IBC security applications in MANET's supported a survey of publications on this subject since the emergence of IBC in 2001. During this paper, we tend to additionally share insights into open analysis issues and denote fascinating future directions during this space.
\end{abstract}

\section{General Terms}

Identity-based Cryptography, Mobile Ad-hoc Networks, OLSR, DSA.

\section{Keywords}

MANET's, IBC, PKI, PKG, CA, DSA

\section{INTRODUCTION}

Research on security of MANET's remains active many years of exploration, in each world and business. It is partially as a result of the very fact that no mature answer is wide accepted and also the growing availableness of little personalized mobile devices with peer to look communication capability through wireless channels. General security needs for MANET's embody [1] knowledge Confidentiality that keeps knowledge secret to intruders, data integrity that stops knowledge from being changed or modified, knowledge freshness that keeps knowledge within the correct order and up-to-date, data availability that ensures knowledge to be obtainable for the asking, knowledge Identity Authentication that verifies that the information or request came from a selected or valid sender and non-repudiation that ensures a node cannot deny causation a message.

Security mechanisms are used in the wide area unit and evidenced to be effective in wired networks. It is not invariably applicable to MANET's. Attacks which will be effectively detected and prevented in wired networks are massive security challenges in MANET's. Examples embody, however are not restricted to any network, identity/address spoofing, message meddling and forgery, message replay etc. As compared to wired networks the combination of the subsequent characteristics of MANET's build it particularly difficult to realize security requirements:

- Lack of a network infrastructure and on-line administration.

- Configuration and node membership dynamics.

- The potential business executive attacks.

Security proposals in early analysis area unit generally attackoriented. They usually initial determine many security threats then enhance the present protocol or propose a replacement protocol to improve them. Such solutions area unit designed expressly against limited attack models. They work well within the presence of limited attack models, however they might collapse below combined or unforeseen attacks [2].

Cryptography is then accustomed offer a general style type framework. Cryptography techniques utilized in MANET's are often classified into two classes, namely as trigonal Key based mostly and uneven Key based. In trigonal key based mostly schemes, if an aggressor compromises the trigonal key of a bunch of users, then all the encrypted messages for that cluster will be exposed and the uneven key based mostly schemes will offer additional functionalities than trigonal ones, e.g., key distribution is far easier, authentication and non-repudiation area unit obtainable, compromise of a nonpublic key of a user will not reveal messages encrypted for alternative users within the cluster. However, they are typically computationally high-ticket. Traditional uneven cryptography wide and effectively utilized in the net depends on a Public Key Infrastructure (PKI). The success of PKI depends on the supply and security of a Certificate Authority (CA), a central management purpose that everyone trusts. Normally MANET's, applying PKIs by maintaining a central management purpose is clearly not invariably feasible. Another obstacle that impedes PKI's employment in MANET's is that the significant overhead of transmission and storage of public key certificates (PKCs).

Identity-based cryptography (IBC) could be a special kind of public key cryptography. It's AN approach to eliminate the requirement of a CA and PKCs. Since 2001, IBC has attracted additional and additional attention from security researchers. Some properties of IBC build it particularly appropriate for MANET's. Fang et al. [3], [4] summarize the benefits of IBC to MANET's:

- $\quad$ Easier to deploy with none infrastructure demand. This protects certificate distribution, whereas delivery - free pair wise keys with none interaction between nodes.

- Its resource needs, relating to method power, storage house, communication information measure, area unit a lot of lower.

- The general public key of IBC is self-proving and might carry a lot of helpful data.

We believe that IBC, with its quick development in recent years, could be a promising answer for Manet security problems. This has driven U.S. to jot down this survey. We have a tendency to gift a comprehensive image and have known the state of the art of important IBC security applications in MANET's by conducting a survey on publications over the recent decade from 2001 to 2010. It also share insights into open analysis issues and indicate attentiongrabbing future directions during this space. Since difficulty of MANET security lies on variations between MANET's and wired infrastructure networks in network and lower layers. Identity-based cryptosystems are principally utilized in 
network layer, i.e. in routing protocols. Hence, most of previous publications specialize in key management and routing protocols. A non-trivial purpose of this survey is that they have a tendency to review the proposals within the literature from a system engineering perspective on however a sensible system works with these existing proposals, e.g. a way to started a secure routing among a collection of nodes. During this perspective, we have a tendency to determine some of the weaknesses of those protocols that can-not be found if we glance at them one by one.

\section{RELATED WORK}

Identity-based cryptography schemes are within the class of Asymmetric Key based cryptography. Identity-based cryptography specifies a cryptosystem during which each public and personal key are supported the identities of the users. The basic idea of IBC was 1st planned by Shamir [5] in 1984. Such a theme has the property that a user's public secret key is simply calculated or easily guessed by the operator of his identity, whereas a user's personal key is calculated for him by a trustworthy authority, called a Private Key Generator (PKG). The identity-based public key cryptosystem is an alternate for certificate-based PKI, particularly once economical key management and moderate security are needed. Compared to ancient PKI, it saves storage and transmission of public keys and certificates that is very enticing for devices forming MANET's. For a protracted time once Shamir revealed his plan, the event on IBC was terribly slow. Joux [6], in 2000, showed that Weil pairing is used for good by mistreatment it during a protocol to construct multilateral one-round Diffie-Hellman key agreement. This was one in all the breakthroughs in key agreement protocols. After this, Boneh and Franklin [7] conferred at Crypto 2001 Associate in Nursing identity-based secret writing theme supported properties of linear pairings on elliptic curves, which is the first absolutely useful, economical and incontrovertibly secure identity-based secret writing theme.

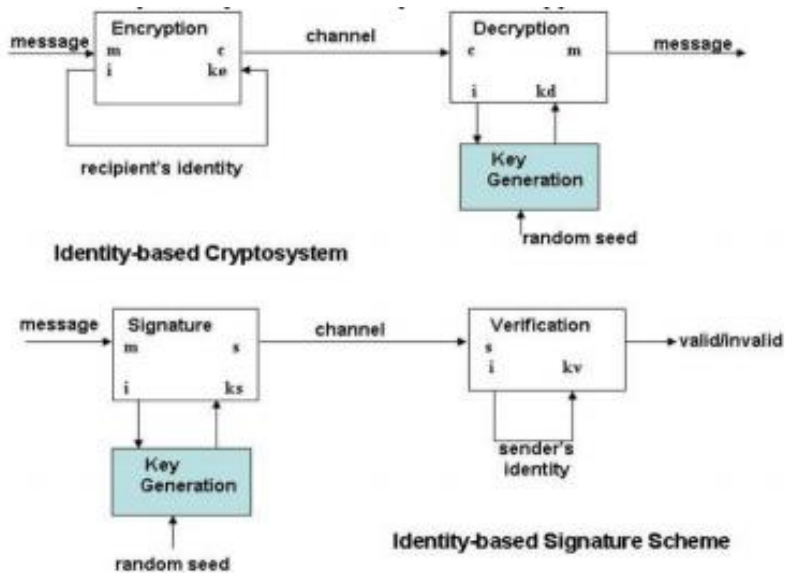

Fig.1: Shamir's identity based cryptosystem and signature scheme

\section{SECURE ROUTING PROTOCOLS USING IBC}

Routing in Manet's permits packet delivery from one node to a different by method of intermediate nodes. It is the fundamental issue thought of in MANET's, so secure routing could be a elementary issue in painter security. Secure routing ensures sure-fire routing among authentic nodes with antagonist nodes existing around or within the network, and forms the bedrock of a secure painter system. A crucial application of IBC in MANET's is to style secure routing protocols. Generally, compared to traditional cryptosystems, IBC provides the subsequent blessings in terms of secure routing; IBC improves potency of secure routing. Once secure keys area unit available, IBC is applied to either on-demand routing protocols like DSR, or link state routing protocols like OLSR. The routing messages encrypted and signed by the sender and decrypted and verified by the receiver exploitation IBC. to shield routing messages, on same security level, IBC encryption/decryption schemes area unit quicker, and IBC signature is shorter.

\section{A. A Security design to Secure OLSR}

Adjih et al. [21] propose a security design to secure OLSR exploitation IBC. Their proposal relies on the work of [20], [8]. In their theme, Associate in Nursing (offline) metallic element is answerable of certifying or distribution keys of every node taking part within the trusty network every node connection the network can have the general public key of the metallic element. This key's denoted the world key. Later, any node getting into the ad-hoc network might diffuse its public keys, with a specific key exchange protocol, with correct parameters and signatures. The key that is employed later to sign message is called the native key, and may be either its international key, or fresh generated private/public keys. A node would begin originating OLSR management messages, sign language them exploitation the native key with a selected extension that pretends a special signature message. Technical details of the theme aren't given within the paper, e.g. however keys area unit generated and distributed, however packets are signed and encrypted.

\section{B. A Key Management Integrated OLSR Routing}

Protocol most routing protocols do not take into account key management problems. In [22], Zhao and Aggarwal propose a secure routing protocol integrated with key management supported previous work of [7], [23], [19], and exploitation planned proactive security approach, they style a secure routing protocol for pre-planned MANET's. The network starts with initial nodes the primary part is routing setup. Initial nodes contact and find system secret from an off-line official administrator. With the system secret, the nodes communicate with one another firmly and originated routing table. The second part is secret update. Since routing is already originated, initial nodes will communicate with every other firmly exploitation pair-wise session key and contribute to a brand new secret. System secret is updated sporadically or when necessary.

Digital Signature: - Digital signatures have always been an integral part of cryptography in history. Cryptography is the study of mathematical techniques related to aspects of information security such as confidentiality, data integrity, entity authentication, and data origin authentication. The security in MANET's is defined as a combination of processes, procedures, and systems used to ensure confidentiality, authentication, integrity, availability, and non repudiation. Digital Signature is a widely adopted approach to ensure the authentication, integrity, and non repudiation of MANET's. It can be generalized as a data string, which associates a message (in digital form) with some originating entity or an electronic analog of a written signature. Digital signature schemes can be mainly divided into the following two categories. 
1) Digital signature with appendix:

The original message is required in the signature verification algorithm. Examples include a digital signature algorithm (DSA).

\section{2) Digital signature with message recovery:}

This type of scheme does not require any other information besides the signature itself in the verification process. Examples include RSA.

\section{APPLICATIONS OF IBC IN SPECIAL-PURPOSE MANET'S}

Besides key management and secure routing, there also are other applications of IBC in special MANET's, such as multidomain or multi-TA coalition networks. These applications aren't relevant to general MANET's, thus we deliberately hop over a lot of the detail within the following.

In [24], Balfe et al. conceive of that in IBC infrastructures, entities from multiple TAs may well be gift inside a bigger coalition structure, with every Ta issuance crypto logic keys to entities in its own security domain supported the work of [25], [26], [11], [27], they propose a light-weight, generic and broadly speaking applicable framework enabling the refreshing of privates keys in coalition-forming things. They signifies their contribution is that the improvement upon the apparent approach of merely distributing new non-public keys by encrypting them mistreatment the previous public keys. The authors claim that their theme is secure and state that the framework is applicable to modify secure inter-operation between entities with totally different trustworthy authorities in dynamic coalition's environments, and is especially wellsuited to coalition forming in computation and bandwidth limited MANET's.

In [28], Li et al. contemplate cross-domain key agreement in multi-domain impromptu networks. They propose a replacement IBC scheme supported multiple PKGs, that is additional appropriate for multi-domain impromptu networks. They assume that there are two PKGs-P KG1 and P KG2 two domains, that share identical system parameters, however have totally different master private keys during this scenario, the theme provides encryption/decryption, sign/verify functions between the two domains.

Cai et al. [29] apply IBC to see collaboration in MANET's. They determine the matter of peer collaboration in impromptu networks, particularly once some peers are autonomous, selfish, or malicious in large-scale, heterogeneous networks. Payment-incited mechanism is Associate in Nursing approach for this drawback, however most existing electronic payment schemes either trust online, interactive authorities, or are too significant for MANET's.

The authors style a light-weight and cheat-resistant micropayment theme to stimulate and compensate cooperative peers that sacrifice their resources to relay packets for alternative peers. They base their work on [30], [7]. Their theme uses identity-based signature and verification mechanisms to realize authentication and non-repudiation of commitment proposal messages and commitment confirmation messages, and uses hash-chain to count knowledge volume transmitted. The authors conducted simulations of their schemes. Through simulation results, they claim that once security and collaboration measures are properly implemented, profitable collaboration may be a preferred strategy for all peers in MANET's; and with profitable collaboration, system utility will increase once peers have maximized their potential profit.

In this section, we've got studied applications of IBC in special-purpose MANET's. These applications are solely applicable to terribly restricted eventualities, and aren't popularly helpful.

\section{A. Security considerations of IBC}

The greatest concern of applying IBC in MANET's is that the reliableness of its security. In [29], L. Cai state that it's still laborious to mention whether or not pairing-based cryptosystems (the thought of IBC) are going to be ready to give satisfactory security and potency because the desired level of security rises. They state that because the security needs increase, the price one has got to buy the additional practicality can increase sharply.

They additionally determine some theoretical concern on the pairing-based systems - the BDHP (bilinear Diffiee-Hellman problem) may be a new drawback that has not been wide studied. it's closely associated with the Diffiee-Hellman drawback (DHP) in the elliptic curve cluster. It follows that if one has Associate in Nursing algorithmic rule for the DHP on the curve, one will right away solve the BDHP further. Therefore it's a supply of concern that security depends on the likely intractableness of the DHP rather than the additional natural and additional extensively studied separate Log drawback (DLP).The author states if a Verheul homomorphism may someday be made, albeit it were made only for the class-VI super singular elliptic curves, that will be enough to render all pairing-based cryptosystems fully insecure. From the literature, it seems that up to currently, Verheul's guess has not been well-tried positive or negative. Moody reviews some of the issues that the safety of elliptic curve cryptosystems are based mostly upon, and discusses well the theory of Verheul (including its generalization), and its consequences. The author tries to generalize Verheul's theorem to additional normal curves. As a conclusion, the author leaves it as Associate in Nursing open question to generalize some sort of Verheul's theorem to normal curves with low embedding degree, and states that this work would need new strategies.

To achieve high security and counter attacks towards IBC, researchers counsel golf shot additional strict restraints on its mathematical basis and selecting the elliptic curve and finite field it uses meticulously. Researches on these security concerns and challenges are going to be the longer term work on IBC schemes and their applications.

\section{CONCLUSION}

In this survey, it tells about the major developments in IBC, and therefore the applications of IBC in MANET's in varied areas. In this survey various fields that are new in the upcoming technology.

It also shows some of the drawbacks and challenges of IBC that impose difficulties on its application to MANET's. In the field of MANET's security IBC has already been wide applied. However, they tend to notice there are several problems unaddressed in these applications. On the above discussion it results there are some advantages and disadvantages of the IBC.

To apply IBC higher in MANET's, we tend to should look into properties of IBC and establish its execs and cons. On the one hand, some properties lend IBC attractions to MANET's non-public keys are short and straightforward to come up with 
and store, public keys are implicitly carried by their identities, therefore there is no got to distribute and store certificates of partners or public key of CA. On the other hand, its alternative properties seem awkward in MANET's. Another issue that is not mentioned there (because there are no thanks to work around it) however a haul for several MANET's is that from the character of IBC, it needs the system parameters be distributed to any or all human activity parties before any messages will be en-crypt/decrypted. This requirement excludes the therefore known as truly ad hoc networks out of its scope. In those networks, a bunch of strangers return together with none central node answerable of the administration and therefore the organization of the network. The key can solely be generated on-line contributively by entrusted peers. Thus, they're inevitably subject to Byzantine attacks, and should be whole appropriated by adversaries. Considering properties on either side of IBC in MANET's, it discover a kind of MANET's that is most fitted for IBC there is associate degree administrator that generates and distributes initial system parameters to any or all nodes. The administrator will authenticate the identity of a node, and assign initial non-public key thereto. For those MANET's that meet these necessities, e.g. detector networks, military networks like moving troopers with wearable computers, moveable communication systems for future public safety, emergency and disaster applications, IBC is that the most promising security answer, but there appear no excellent solutions however.

Future scope related to this survey is that there are some security issues, concerns and challenges that will be improved in IBC. It also provide the confidentiality to the valid user with authentication, no intruder can break IBC confidentiality.

\section{REFERENCES}

[1] L. Abusalah, A. Khokhar, and M. Guizani, "A survey of secure mobile ad hoc routing protocols," IEEE Commun. Surveys \& Tutorials, IEEE,vol. 10, no. 4, pp. 78-93, 2008.

[2] H. Yang, H. Luo, F. Ye, S. Lu, and L. Zhang, "Security in mobile adhoc networks: challenges and solutions," IEEE Wirel. Commun., vol. 11,no. 1, pp. 38-47, 2004.

[3] Y. Fang, X. Zhu, and Y. Zhang, "Securing resourceconstrained wireless ad hoc networks," Wireless Commun., vol. 16, no. 2, pp. 24-29, 2009.

[4] Y. Zhang, W. Liu, W. Lou, and Y. Fang, "Securing mobile ad hoc networks with certificateless public keys," IEEE Trans. Dependable Secur. Comput. vol. 3, no. 4, pp. 386-399, 2006.

[5] A. Shamir, "Identity-based cryptosystems and signature schemes" in Proc. Crypto 1984, 1984.

[6] A. Joux, "A one round protocol for tripartite diffiehellman," in ANTS IV, ser. LNCS, vol. 1838. SpringerVerlag, 2000, pp. 385-394.

[7] Boneh and Franklin, "Identity-based encryption from the weil pairing," in Proc. Crypto 2001, ser. LNCS, vol. 2139. Springer, 2001, pp. 213-219.

[8] D. Boneh, B. Lynn, and H. Shacham, "Short signatures from the weil pairing," in Proc. ASIACRYPT, ser. LNCS, vol. 2248. Springer-Verlag, 2001, pp. 514-532.
[9] R. Dutta, R. Barua, and P. Sarkar, "Pairing-based cryptographic protocols: A survey," Cryptology ePrint Archive, Report 2004/064, Jun. 242004.

[10] A. Shamir, "How to share a secret," Communications of the ACM, vol. 22, no. 11, 1979.

[11] A. Khalili, J. Katz, and W. A. Arbaugh, "Toward secure key distribution in truly ad-hoc networks," in SAINT Workshops. IEEE Computer Society, 2003, pp. 342-346.

[12] R. Bobba, L. Eschenauer, V. Gligor, and W. Arbaugh, "Bootstrapping security associations for routing in mobile ad-hoc networks," in IEEE Global Telecommunications Conference 2003. IEEE Computer Society Press, 2003.

[13] H. Deng, A. Mukherjee, and D. P. Agrawal, "Threshold and identitybased key management and authentication for wireless ad hoc networks," in ITCC (1). IEEE Computer Society, 2004, pp. 107-111.

[14] H. Deng and D. P. Agrawal, "TIDS: threshold and identity-based security scheme for wireless ad hoc networks," Ad Hoc Networks, vol. 2, no. 3, pp. 291-307, 2004.

[15] P. Xia, M. Wu, K. Wang, and X. Chen, "Identity-Based Fully Distributed Certificate Authority in an OLSR MANET," in 4th Wireless Communications, Networking and Mobile Computing. IEEE, 2008, pp. 1-4.

[16] J. V. D. MERWE, D. DAWOUD, and S. McDONALD, "A survey on peer-to-peer key management for mobile ad hoc networks," ACM Comput.Surv., vol 39,no 1,pp. 145,2007 .

[17] S.Xu and S.Capkun, "Distributed and secure bootstrapping of mobile ad hoc networks:Framework and constructions,"ACM Trans. Inf.Syst.Secure.,vol.12,no. 1.pp. 1-37,2008.

[18] S.Zaho and Aggarwal, "Againstmobile attacks in ad hoc networks," in proc. IEEE International Conferences on Information Theory and Information Security, 2010.

[19] X. Boyen, "Multipurpose identity-based signcryption: A swiss army knife for identity-based cryptography," in Proc. Crypto 2003, 2003.

[20] J. Cha and J. Cheon, "An identity-based signature from gap diffie-hellman groups," in PKC: International Workshop on Practice and Theory in Public Key Cryptography, vol. 2567. LNCS, 2003.

[21] C. Adjih, D. Raffo, and P. Muhlethaler, "Attacks against olsr: Distributed key management for security," in Proc. OLSR Interop and Workshop, 2005.

[22] S. Zhao and A. Aggarwal, "PAPA-UIC: a design approach and a frame-work for secure mobile ad hoc networks," Security and Communication Networks, John Wiley \& Sons, vol. 1, pp. 371-383, 2010.

[23] B. Lynn, "Authenticated identity-based encryption," Cryptology ePrint Archive, Report 2002/072, Jul. 11 2002.

[24] S. Balfe, K. D. Boklan, Z. Klagsbrun, and K. G. Paterson, "Key refreshing in identity-based cryptography and its applications in MANET's," in Military Communications Conference, 2007. MILCOM 2007. IEEE. IEEE, 2007, pp. 1-8. 
[25] K. Hoeper and G. Gong, "Bootstrapping security in mobile adhoc networks using identity-based schemes with key revocation," University of Waterloo, Report 2006-04, 2006. [Online]. Available http://www.comsec.uwaterloo.ca/ khoeper/IBCrevocatio n_hoeper.pdf

[26] D. Carman, "New directions in sensor network key management," International Journal of Distributed Sensor Networks, vol. 1, pp. 3-15, 2004.

[27] S. Balfe, K. D. Boklan, Z. Klagsbrun, and K. G. Paterson, "Toward hierarchical identity-based cryptography for tactical networks," inMili-tary Communications Conference, 2004. MILCOM 2004. IEEE. IEEE, 2004, pp. 1-8.
[28] F. Li, Y. Hu, and C. Zhang, "An identity-based signcryption scheme for multi-domain ad hoc networks," in Proc. 5th international conference on Applied Cryptography and Network Security. Springer-Verlag, 2007, pp. 373-384.

[29] L. Cai, J. Pan, X. Shen, and J. W. Mark, "Peer collaboration in wireless ad hoc networks," in Proc. 4th International IFIP-TC6 Networking Conference, ser. LNCS, vol. 3462. Springer, 2005, pp. 840-852.

[30] G. Gentry and A. Silverberg, "Hierarchical ID-based cryptography," in Proc. ASIACRYPT. LNCS, SpringerVerlag, 2002 